\title{
Arterial secondary blood flow patterns visualized with vector flow ultrasound
}

Pedersen, Mads Møller; Pihl, Michael Johannes; Hansen, Jens Munk; Hansen, Peter Møller; Haugaard, Per; Nielsen, Michael Bachmann; Jensen, Jørgen Arendt

\section{Published in:}

Proceedings of the 2011 IEEE International Ultrasonics Symposium

Link to article, DOI:

10.1109/ULTSYM.2011.0306

Publication date:

2011

Link back to DTU Orbit

Citation (APA):

Pedersen, M. M., Pihl, M. J., Hansen, J. M., Hansen, P. M., Haugaard, P., Nielsen, M. B., \& Jensen, J. A. (2011). Arterial secondary blood flow patterns visualized with vector flow ultrasound. In Proceedings of the 2011 IEEE International Ultrasonics Symposium (pp. 1242-1245). IEEE. https://doi.org/10.1109/ULTSYM.2011.0306

\section{General rights}

Copyright and moral rights for the publications made accessible in the public portal are retained by the authors and/or other copyright owners and it is a condition of accessing publications that users recognise and abide by the legal requirements associated with these rights.

- Users may download and print one copy of any publication from the public portal for the purpose of private study or research.

- You may not further distribute the material or use it for any profit-making activity or commercial gain

- You may freely distribute the URL identifying the publication in the public portal 


\title{
Arterial secondary blood flow patterns visualized with vector flow ultrasound
}

\author{
Mads Møller Pedersen ${ }^{1,2}$, Michael Johannes Pihl ${ }^{2}$, Jens Munk Hansen ${ }^{2}$, \\ Peter Møller Hansen ${ }^{1,2}$, Per Haugaard ${ }^{3}$, Michael Bachmann Nielsen ${ }^{1}$ and Jørgen Arendt Jensen ${ }^{2}$ \\ ${ }^{1}$ Dept. of Radiology, Copenhagen University Hospital, Rigshospitalet, DK-2100 Copenhagen, Denmark \\ ${ }^{2}$ Center for Fast Ultrasound Imaging, Dept. of Elec. Eng., Bldg. 349 \\ Technical University of Denmark, DK-2800 Lyngby, Denmark \\ ${ }^{3}$ R\&D Applications \& Technologies, BK Medical, DK-2730 Herlev, Denmark
}

\begin{abstract}
This study presents the first quantification and visualization of secondary flow patterns with vector flow ultrasound. The first commercial implementation of the vector flow method Transverse Oscillation was used to obtain in-vivo, 2D vector fields in real-time. The hypothesis of this study was that the rotational direction is constant within each artery. Three data sets of 10 seconds were obtained from three main arteries in healthy volunteers. For each data set the rotational flow patterns were identified during diastole. Each data set contains a $2 \mathrm{D}$ vector field over time using the vector angles and velocity magnitudes the blood flow patterns were visualized using streamlines in Matlab (Mathworks, Natick, MA, USA). The rotational flow was quantified by the angular frequency for each cardiac cycle, and the mean rotational frequencies and standard deviations were calculated for the abdominal aorta $\{-1.3 \pm 0.4 ;-1.0 \pm 0.3 ;-0.9 \pm 0.2\} \mathrm{Hz}$, the common iliac artery $\{-0.4 \pm 0.1 ;-1.0 \pm 0.2 ;-0.4 \pm 0.1\} H z$, and the common carotid artery $\{0.8 \pm 0.3 ; 1.4 \pm 0.3 ; 0.4 \pm 0.1\}$ Hz. A positive sign indicates an anti-clockwise rotation, and a negative sign indicates clockwise rotation. The sign of the rotational directions within each artery were constant.
\end{abstract}

\section{INTRODUCTION}

The vector flow technique, Transverse Oscillation (TO), has been investigated thoroughly since the first presentations in 1996 [1]-[4]. The method has been tested in simulation [5], by in-vivo studies with the experimental scanner, RASMUS [6], and in comparison with magnetic resonance (MR) angiography [7]. In contrast to conventional Doppler techniques, TO directly provides the absolute velocities and flow angles of blood at any angle in a 2D vector field. The method is non-invasive, as it does not require contrast agents.

Measurement of the transverse velocity component has been investigated with several other techniques. Particle-image velocimetry (PIV) obtains the velocity of moving particles using an optical system, where the particles are illuminated with pulsed light. The movement of the speckles are tracked to form a vector field [8]. Replacing the light and photo equipment with ultrasound, the technique echo PIV uses micro bubbles as contrast agent. In a phantom study the flow patterns in stenosis and aneurysm phantoms clearly illustrates the flow patterns [9]. The velocity estimation of echo PIV was recently correlated with phase contrast magnetic resonance (PCMRI) with good agreement in a phantom study [10]. An in vivo study of the carotid artery in live rats, showed a deviation

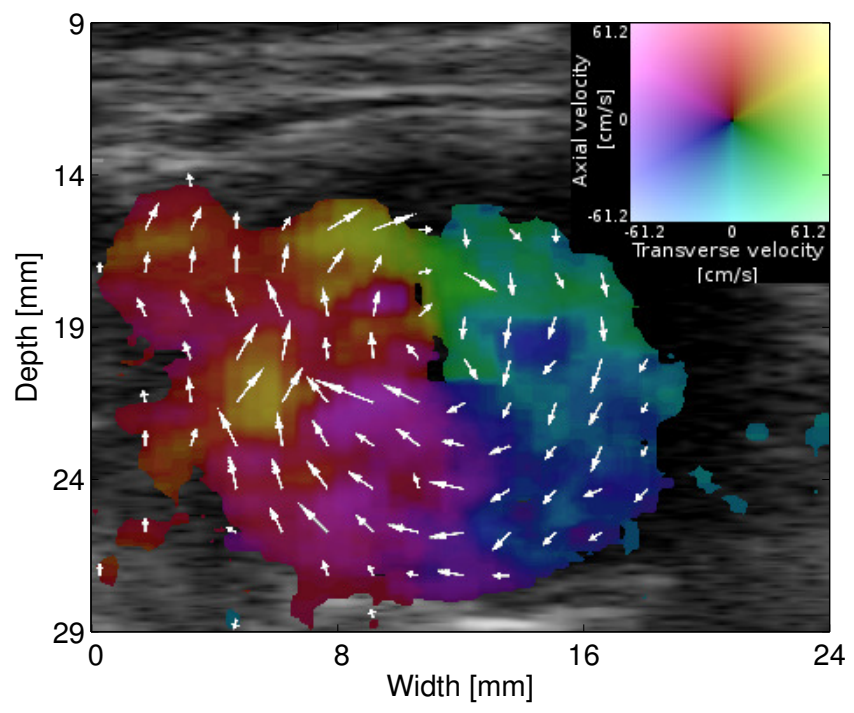

Fig. 1: Real-time, in vivo vector flow ultrasound image of the abdominal aorta in the transverse plane. The vector velocity color map is shown in the upper right corner.

of $15 \%$ from the Doppler-measured peak velocities [11]. The vortex flow of the human heart was quantified with PIV using micro bubbles as contrast agent [12].

Color Doppler has been used to illustrate the flow patterns in the aorta. Based on the color patterns of in vivo transesophageal ultrasound, it was concluded that blood rotates anticlockwise in the diastole as a result of backflow [13]. A comment on this finding suggested that such color patterns might simply be a result of a $20^{\circ}$ misalignment of the transverse plane [14], as only the axial velocities were measured.

PCMRI techniques are able to visualize and quantify secondary flow patterns [15]. The MR vector information makes it possible to estimate physiological forces, such as wall shear stress [16], [17].

With the recent implementation of TO in a commercial scanner, detailed real-time blood velocity magnitudes and flow directions have been illustrated in-vivo [18], and have been compared to spectral velocity estimation [19]. The presence 
(a) Systole $(0.0 \mathrm{~s})$

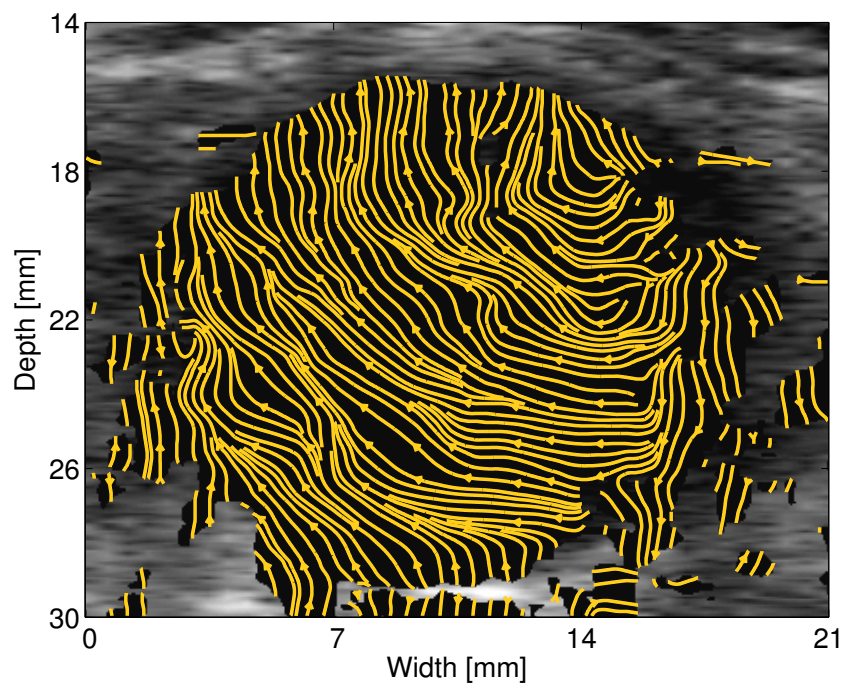

(c) Diastole (0.4 s)

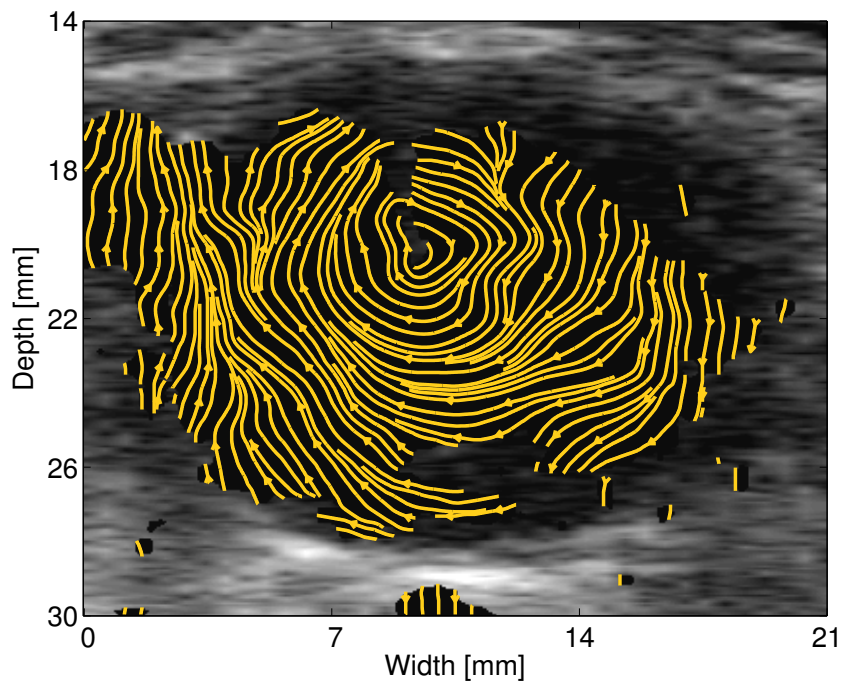

(b) Diastole (0.3 s)

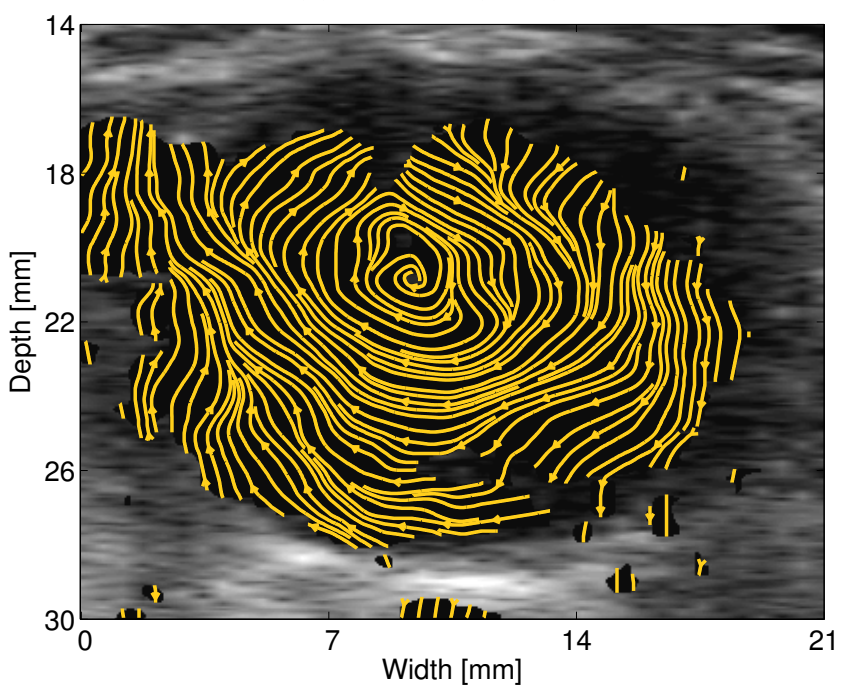

(d) Diastole (0.5 s)

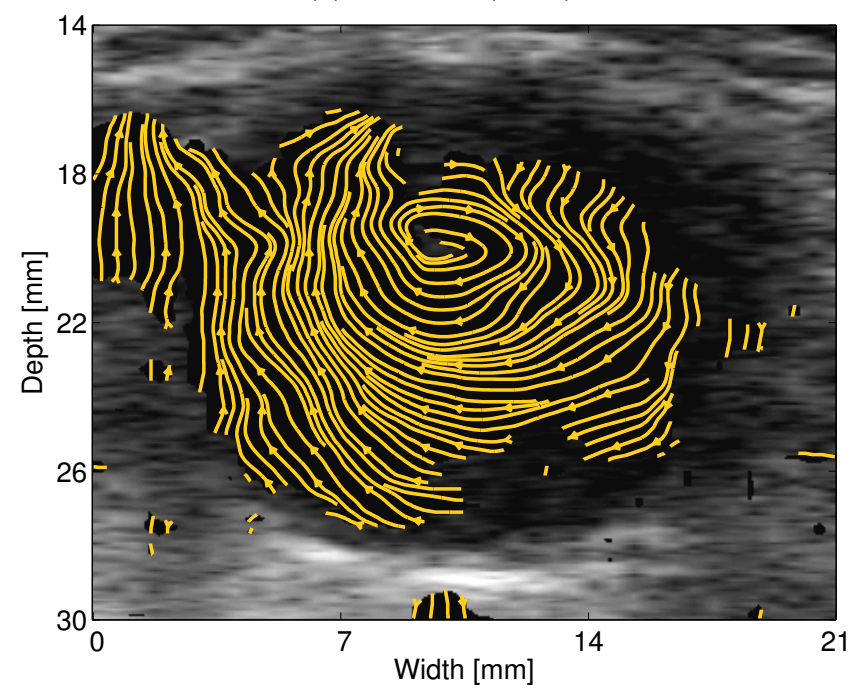

Fig. 2: Vector flow ultrasound of the abdominal aorta in the transverse plane. The streamlines through one cardiac cycle illustrate a systolic flow pattern (a) with no secondary flow, whereas the pattern is fully developed during the diastole (b-c).

of disturbed flow patterns in the carotid bifurcation have been confirmed visually and quantitatively [6], [7]. Thus, rotational flow patterns are visualized and can be obtained in realtime for further analysis. Previous studies have indicated that rotational flow patterns may play a role in the pathogenesis of atherosclerosis [20], [21].

With this study the rotational flow patterns are visualized with in vivo, real-time vector flow ultrasound for the first time. Furthermore, a quantitative method is presented which calculates the rotational frequency.

\section{MethodS}

\section{A. Data acquisition}

Five healthy volunteers participated in this study. For each volunteer the transverse plane of three arteries were scanned with vector flow ultrasound: The abdominal aorta (AA), the common carotid artery (CCA), and the common iliac artery (CIA). A commercial vector flow ultrasound scanner (ProFocus Ultraview, BK Medical, Denmark) was used with a $5 \mathrm{MHz}$ linear array (type: 8670, BK Medical, Denmark) and prototype scanner software. Ten seconds of data were obtained as AVI files with a mean frame rate of $21 \pm 5 \mathrm{~Hz}$ with a range of [13 28] Hz. Three data sets for each artery, which presented a rotational flow pattern, were selected, resulting in nine data sets. A frame example of the real-time colour encoded vector data is shown in Fig. 1.

\section{B. Real-time vector data}

The real-time vector information is displayed as colours and by post processing the axial, $v_{z}$, and transverse, $v_{x}$, vector 
velocity magnitudes were obtained through time in $2 \mathrm{D}$ vector fields. Further details on data processing of the colour encoded vector information is provided in [22].

\section{Streamline imaging}

Streamlines were used to visualize the blood flow patterns for every frame. The line density was set low to make the visual impression as clear as possible. In Fig. 2 the development of a secondary flow pattern through the cardiac cycle is shown for the abdominal aorta.

\section{Angular speed and frequency of a $2 D$ vector field}

A grid defining subareas in the $2 \mathrm{D}$ vector field is set from the center of the rotation and the rotational frequency of each subarea is calculated [23] by

$$
(\nabla \times F)_{z}=\frac{\partial}{\partial x} F_{y}-\frac{\partial}{\partial y} F_{x}
$$

For each data set, the frequencies during each diastolic phase were found and a mean rotational frequencies and a standard deviation was calculated for each dataset. The frequencies were calculated using Matlab.

\section{RESUlTS AND Discussion}

Nine data sets were acquired and post processed to calculate the mean angular frequencies and standard deviations of each data set. The results are shown in Fig. 4.

All frequencies of AA and CIA were negative, indicating a unidirectional, clockwise rotation. All CCA frequencies were positive, indicating a unidirectional, anticlockwise rotation.

TO is depending on the pulse repetition frequency and wall filter settings and were optimised to obtain low velocities. Thus, the high velocity flow during systole may have caused aliasing, thus not providing the true flow pattern. Both MR [15] and color flow studies [13] have illustrated a rotational flow during systole. Thus, rotational flow patterns during systole should be addressed in future TO studies.

With the present setup, the mean frame rate was limited to $21 \mathrm{~Hz}$. An increase in the temporal resolution would provide further details on the development of the rotational flow patterns and the peak frequencies during the cardiac cycle.

The angular frequencies and streamlines imposed on the Bmode image are shown for one image frame of the abdominal aorta in Fig. 3. The center of the rotation represents the maximum angular frequency of $-1.32 \mathrm{~Hz}$, whereas the peripheral magnitudes are lower. If the image represented a water tank, a paddle placed in a peripheral subarea would rotate at a lower frequency compared to a paddle placed in the center. The streamlines in combination with the angular frequencies and the B-mode image are suggested for clinical imaging of rotational flow with TO.

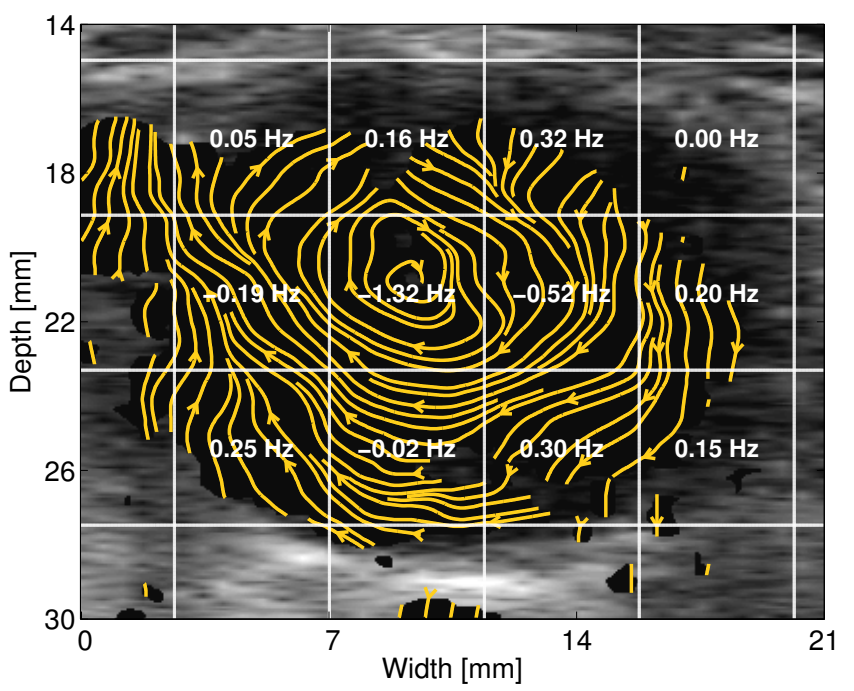

Fig. 3: In a transverse section of the abdominal aorta, the angular frequency of each subarea are imposed on the streamlines and the B-mode image.

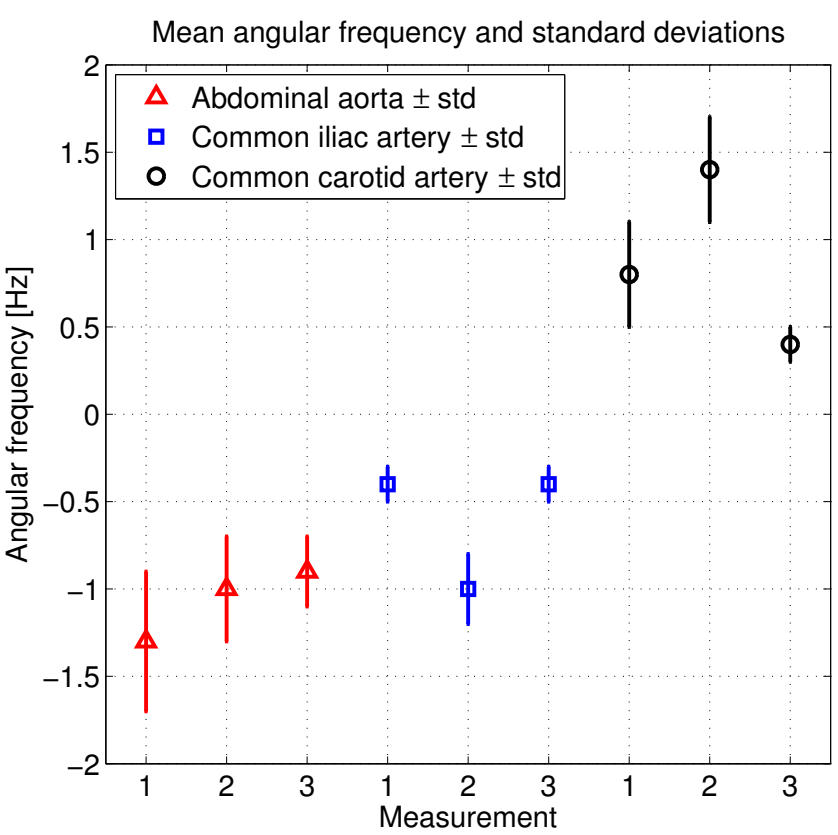

Fig. 4: The angular frequencies, mean values, and standard deviations for all measurements. The direction is given by the sign, which is identical for all measurements of each artery. 


\section{CONCLUSION}

With the commercial real-time vector flow ultrasound scanner, in vivo data can be used to calculate the angular frequencies. Secondary flow patterns have been measured in diastole in the AA, CIA, and CCA. The diastolic secondary flow in AA and CIA rotates clockwise, whereas CCA rotates anticlockwise.

A new plot presenting both the flow streamlines, the angular frequencies, and the B-mode image was suggested for clinical use. In conclusion real-time vector flow can visualize and quantify secondary flow patterns of three main arteries and the rotational directions were constant within each artery.

\section{ACKNOWLEDGEMENTS}

This work was supported by grant 26-04-0024 from the Danish Science Foundation and by BK Medical Aps, Denmark.

\section{REFERENCES}

[1] P. Munk, "Estimation of the 2-D flow vector in ultrasonic imaging: a new approach," Master's thesis, Department of Information Technology, Technical University of Denmark, 1996.

[2] J. A. Jensen and P. Munk, "A New Method for Estimation of Velocity Vectors," IEEE Trans. Ultrason., Ferroelec., Freq. Contr., vol. 45, pp. 837-851, 1998.

[3] P. Munk, "Estimation of blood velocity vectors using ultrasound," Ph.D. dissertation, Department of Information Technology, Technical University of Denmark, Lyngby, Denmark, 2000.

[4] J. A. Jensen, "A New Estimator for Vector Velocity Estimation," IEEE Trans. Ultrason., Ferroelec., Freq. Contr., vol. 48, no. 4, pp. 886-894, 2001.

[5] J. Udesen and J. A. Jensen, "Investigation of Transverse Oscillation Method," IEEE Trans. Ultrason., Ferroelec., Freq. Contr., vol. 53, pp. 959-971, 2006.

[6] J. Udesen, M. B. Nielsen, K. R. Nielsen, and J. A. Jensen, "Examples of in-vivo blood vector velocity estimation," Ultrasound Med. Biol., vol. 33, pp. 541-548, 2007.

[7] K. L. Hansen, J. Udesen, N. Oddershede, L. Henze, C. Thomsen, J. A Jensen, and M. B. Nielsen, "In vivo comparison of three ultrasound vector velocity techniques to $\mathrm{mr}$ phase contrast angiography," Ultrasonics, vol. 49, pp. 659-667, 2009.

[8] R. J. Adrian, "Particle-imaging techniques for experimental fluid mechanics," Annu. Rev. Fluid Mech., vol. 23, pp. 261-304, 1991.

[9] L. Liu, H. Zheng, L. Williams, F. Zhang, R. Wang, J. Hertzberg, and R. Shandas, "Development of a custom-designed echo particle image velocimetry system for multi-component hemodynamic measurements: system characterization and initial experimental results," Phys. Med. Biol., no. 53, pp. 1397-1412, 2008.
[10] F. Zhang, C. Lanning, L. Mazzaro, A. J. Barker, P. E. Gates, W. D. Strain, J. Fulford, O. E. Gosling, A. C. Shore, N. G. Bellenger, B. Rech, J. Chen, J. Chen, and R. Shandas, "In vitro and preliminary in vivo validation of echo particle velocimetry in carotid vascular imaging," Ultrasound Med. Biol., vol. 37, no. 3, pp. 450-464, 2011.

[11] L. Niu, M. Qian, K. Wan, W. Yu, Q. Jin, T. Ling, S. Gao, and H. Zheng, "Ultrasonic particle image velocimetry for improved flow gradient imaging: algorithms, methodology and validation," Phys. Med. Biol., no. 55, pp. 2103-2120, 2010.

[12] G. R. Hong, G. Pedrizzetti, G. Tonti, P. Li, Z. Wei, J. K. Kim, A. Baweja, S. Liu, N. Chung, H. Houle, J. Narula, and M. A. Vannan, "Characterization and quantification of vortex flow in the human left ventricle by contrast echocardiography using vector particle image velocimetry," JACC Cardiovasc Imaging, vol. 1, no. 6, pp. 705-717, 2008.

[13] L. J. Frazin, G. Lanza, M. Vonesh, F. Khasho, C. Spitzzeri, S. McGee K. B. C. D. Mehlman, J. Talano, and D. McPherson, "Functional chiral asymmetry in descending thoracic aorta," Circulation, vol. 82, no. 6, pp. 1985-1994, 1990.

[14] J. D. Thomas, "Flow in the descending aorta. a turn of the screw or a sideways glance?" Circulation, vol. 82, no. 6, pp. 2263-2265, 1990.

[15] P. J. Kilner, G. Z. Yang, R. H. Mohiaddin, D. N. Firmin, and D. B. Longmore, "Helical and retrograde secondary flow patterns in the aortic arch studied by three-directional magnetic resonance velocity mapping," Circulation, vol. 88, no. 5 Pt 1, pp. 2235-47, Nov. 1993.

[16] E. T. Bieging, A. Frydrychowicz, A. Wentland, B. R. Landgraf, K. M. Johnson, O. Wieben, and C. J. François, "In vivo three-dimensional mr wall shear stress estimation in ascending aortic dilatation," J Magn Reson Imaging, vol. 33, no. 3, pp. 589-97, Mar. 2011.

[17] M. Markl, W. Wallis, and A. Harloff, "Reproducibility of flow and wall shear stress analysis using flow-sensitive four-dimensional mri," J Magn Reson Imaging, vol. 33, no. 4, pp. 988-94, Apr. 2011.

[18] P. M. Hansen, M. M. Pedersen, K. L. Hansen, M. B. Nielsen, and J. A. Jensen, "New technology - demonstration of a vector velocity technique," Ultraschall in der Medizin/European Journal of Ultrasound, vol. 32, pp. 213-5, 2011.

[19] M. M. Pedersen, M. J. Pihl, P. Haugaard, J. M. Hansen, K. L. Hansen, M. B. Nielsen, and J. A. Jensen, "Comparison of real-time in-vivo spectral and vector velocity estimation," Ultrasound Med. Biol., p. Submitted, 2011.

[20] A. E. Hugh and J. A. Fox, "The precise localisation of atheroma and its association with stasis at the origin of the internal carotid artery-a radiographic investigation.” $\mathrm{Br} J$ Radiol, vol. 43, no. 510, pp. 377-383, 1970.

[21] V. Fuster, B. Stein, J. A. Ambrose, L. Badimon, J. J. Badimon, and J. H. Chesebro, "Atherosclerotic plaque rupture and thrombosis. evolving concepts." Circulation, vol. 82, no. 3 Suppl, pp. 47-59, 1990.

[22] M. M. Pedersen, M. J. Pihl, P. Haugaard, M. B. Nielsen, and J. A. Jensen, "Preliminary quantification of complex blood flow using real-time in vivo vector flow ultrasound," in IEEE Proc., 2010, pp. 1088-1091.

[23] B. Lautrup, Physics of continuous matter: Exotic and everyday phenomena in the macroscopic world. Taylor \& Francis, 2005. 